\section{A study of heredity as a risk factor in strabismus}

\section{Abstract}

Aims Inheritance is recognised to have a part in the aetiology of strabismus but previous studies have not adequately distinguished between different types of strabismus leading to wide variations in reported findings. The aim of this study was to investigate the importance of heredity in different types of strabismus.

Methods The parents of children attending for treatment of strabismus over a one-month period were interviewed to identify relatives with a history of strabismus. A complete three-generation pedigree was established for 96 index cases who were classified into four groups: infantile esotropia (26 cases), accommodative esotropia (49 cases), anisometropic esotropia (15 cases), and exotropia (six cases).

Results Forty-three of a total of 165 (26.1\%) first degree relatives of patients with hypermetropic accommodative esotropia were affected. In contrast, 15 of a total of 101 $\mathbf{( 1 4 . 9 \% )}$ first degree relatives of patients with infantile esotropia, eight of a total of 66 $\mathbf{( 1 2 . 1 \% )}$ first degree relatives of patients with anisometropic esotropia, and one of a total of 25 (4\%) first degree relatives of patients with exotropia were affected. Analysing the data using logistic regression with a random term for family showed a significantly higher proportion of affected first degree relatives in the accommodative group than in any of the other three diagnostic groups.

Conclusion A history of strabismus appears to be more common in hypermetropic accommodative esotropia than in infantile esotropia, anisometropic esotropia or exotropia. More detailed investigation of the role of heredity in the aetiology of accommodative esotropia is needed. Eye (2002) 16, 519-521. doi:10.1038/ sj.eye. 6700138

Keywords: strabismus; heredity
NG Ziakas, G Woodruff, LK Smith

and JR Thompson

Since Hippocrates ${ }^{1}$ first observed that strabismus could be transmitted from parent to child, ophthalmologists have been intrigued by evidence that some forms of strabismus seem to have a genetic basis. Several population studies have shown a higher prevalence of strabismus among families of an affected individual than among the general population. However, considerable differences have been encountered in these studies.

Sclossmann and Priestley ${ }^{2}$ found that $47.5 \%$ of their patients with strabismus, $48.9 \%$ with esotropia and $36.8 \%$ with exotropia, belonged to families with two or more affected members. Aurell ${ }^{3}$ found that $17.6 \%$ of babies born into families with a first degree relative affected by convergent strabismus developed constant or intermittent esotropia by age of 6 years. Other studies ${ }^{4,5}$ have reported the familial incidence of strabismus to be as high as $65 \%$.

Such wide variations in reported findings are attributable to several factors. A major problem is the heterogeneity of the populations studied. Strabismus is frequently dealt with as a single phenotype failing to distinguish specific subtypes of strabismus that may have entirely different aetiology. This study was undertaken to assess heredity as a risk factor in different types of strabismus.

\section{Materials and methods}

Two research clerks were trained by a genetics nurse to administer a questionnaire about family history. The parents of children under 12 years old attending the children's eye clinic for treatment of strabismus over a one-month period were approached in the clinic waiting room, and consent for participation in the study obtained. A three-generation pedigree was constructed including the child's
Department of

Ophthalmology

University of Leicester Leicester Royal Infirmary

Clinical Sciences Building PO Box 65

Leicester LE2 7LX, UK

Correspondence:

NG Ziakas

University Eye Clinic 93 Metropoleos Street

Thessaloniki 54622

Greece

Tel: +30-31280260

Fax: $+30-31240666$

E-mail: ziakas@

med.auth.gr

Presented at the XII

Congress of the European Society of Ophthalmology, Stockholm, Sweden, June 1999 
grandparents, parents, uncles and aunts, siblings and first cousins. Relatives were classified as being affected if they were reported by the informant to have a definite misalignment of the eyes or to have had squint surgery in the past. The parents were invited to check information on the pedigree and they were contacted again to ensure accuracy of the information recorded. Data of one hundred index cases with a complete family pedigree were collected. Four cases were excluded from analysis because of absence of strabismus/pseudostrabismus (1), microtropia (1), and strabismus associated with other pathology, PHPV (1) and Coats' disease (1).

A total of 96 pedigrees were analysed. Using the orthoptic and medical records, the index cases were classified into four groups: Infantile Esotropia, Accommodative Esotropia, Anisometropic Esotropia, and Exotropia. Patients were classified as infantile esotropia if the esodeviation had arisen in the first 6 months of life and there was less than three dioptres of refractive error in both eyes. Accommodative esotropia was defined either on the basis of a hypermetropic refractive error of greater than +3.0 dioptres of sphere (DS) in both eyes with partial or complete elimination of the deviation with spectacle correction, or on the basis of a high accommodative convergence/accommodation (AC/A) ratio. Anisometropic esotropia was defined as esotropia associated with a difference in refractive error between the two eyes of greater than, or equal to, 1.5 DS or 1.5 dioptres of cylinder (DC), but less than or equal to +2.50 DS in the more emmetropic eye. Patients with any manifest exodeviation at near or distance were categorised into the exotropia group.

The proportions of affected relatives in the four diagnostic categories were compared using logistic regression with the addition of a random term representing the extended families. The method, devised by Breslow and Clayton, ${ }^{6}$ is implemented in the GLMM procedure of Genstat. ${ }^{7}$ This random term allows for the possibility that people from the same extended family may have correlated responses caused by factors such as shared genes, shared environmental exposures or differences in reporting.

\section{Results}

The distribution of the 96 index cases into the four groups was 26 cases of infantile esotropia, 49 cases of accommodative esotropia, 15 cases of anisometropic esotropia, and six cases of exotropia. All 49 cases of accommodative esotropia were of refractive aetiology and none was due to high $\mathrm{AC} / \mathrm{A}$ ratio. No patients in this series had non-accommodative, non-infantile, nonanisometropoic strabismus.

Thirty-three of the 49 cases $(67.3 \%)$ with accommodative esotropia had at least one affected first degree relative, while 11 of the 26 cases (42.3\%) with infantile esotropia, five of the 15 cases (33.3\%) with anisometropic esotropia, and one of the six cases $(16.6 \%)$ with exotropia had an affected first degree relative.

The total numbers of affected first, second and third degree relatives for each category of index case are given in Table 1. Forty-three of a total of 165 first degree relatives $(26.1 \%)$ of patients with accommodative esotropia were affected, in contrast with 15 of a total of 101 first degree relatives (14.9\%) of patients with infantile esotropia, eight of a total of 66 first degree relatives $(12.1 \%)$ of patients with anisometropic esotropia, and one of a total of 25 first degree relatives $(4 \%)$ of patients with exotropia. Logistic regression gave the estimated odds ratio shown in Table 2. The proportion of affected first degree relatives was significantly higher in the accommodative group than in any of the other three diagnostic groups. There were no significant differences between the other three categories. Similar analyses for second and third degree relatives showed no significant differences and much smaller odds ratios ranging from 0.4 to 1.5 . On average, $18.8 \%$ of first degree relatives of the index cases were affected, while the corresponding figures for second and third degree relatives was $9 \%$ and $5.6 \%$ respectively.

\section{Discussion}

The incidence of strabismus within the general population is considered to be $2.5-4 \% .^{8-10}$ Several studies have shown a much higher prevalence $e^{2-5}$ of strabismus in families with strabismic members, suggesting a genetic component. Subsequently, various modes of inheritance have been proposed that fit some, but not all, familial distributions. ${ }^{2,11,12}$ Most recently, a multifactorial model of transmission ${ }^{13,14}$ has been advocated as the more likely form of inheritance for primary concomitant strabismus. Identification of risk factors within the multifactorial model of inheritance requires separate classification of different types of strabismus in which genetic factors may play different parts. While a slightly greater familial association for esotropia as compared to exotropia has been previously suggested, ${ }^{2}$ there is very little in the literature to support this, and the contribution of different types of esotropia to this observation has not been investigated.

We found a statistically significant risk for 
Table 1 Affected first, second and third degree relatives for each type of strabismus

\begin{tabular}{|c|c|c|c|c|c|c|c|c|c|}
\hline & \multicolumn{3}{|c|}{ 1st degree relatives } & \multicolumn{3}{|c|}{2 nd degree relatives } & \multicolumn{3}{|c|}{$3 \mathrm{rd}$ degree relatives } \\
\hline & Affected & Total & $\%$ & Affected & Total & $\%$ & Affected & Total & $\%$ \\
\hline Infantile esotropia & 15 & 101 & 14.9 & 28 & 250 & 11.2 & 13 & 213 & 6.1 \\
\hline Accommodative esotropia & 43 & 165 & 26.1 & 35 & 466 & 7.5 & 18 & 378 & 4.8 \\
\hline Anisometropic esotropia & 8 & 66 & 12.1 & 11 & 145 & 7.6 & 10 & 141 & 7.1 \\
\hline Exotropia & 1 & 25 & 4.0 & 9 & 58 & 15.5 & 4 & 66 & 6.1 \\
\hline
\end{tabular}

Table 2 Estimated odds ratios (column to row) and 95\% confidence intervals for the comparison of the proportions of affected first degree relatives in the four diagnostic groups

\begin{tabular}{|c|c|c|c|c|}
\hline & Anisometropic esotropia & Accommodative esotropia & Exotropia & Infantile esotropia \\
\hline \multicolumn{5}{|l|}{ Anisometropic esotropia } \\
\hline Accommodative esotropia & $0.4(0.2,0.9)$ & & & \\
\hline Exotropia & $3.2(0.4,27.0)$ & $8.4(1.1,63.8)$ & & \\
\hline Infantile esotropia & $0.8(0.3,2.0)$ & $2.0(1.0,3.9)$ & $0.2(0.0,1.9)$ & \\
\hline
\end{tabular}

strabismus in first degree relatives of patients with hypermetropic accommodative esotropia that was not present for second or third degree relatives, or for any other strabismic group in our series.

Because our index cases were drawn from patients with strabismus attending a children's eye clinic, the proportion of each type of strabismus in the 96 cases will differ from that found in the general population. While treatment and referral patterns will have affected the proportion of the different types of strabismus amongst our index cases, there is no reason to think that these factors would explain why the parents of children with accommodative squint were more often able to identify a first degree relative with squint than the parents of children with other kinds of strabismus.

We interviewed the parents of 96 index cases and identified 2074 relatives in whom we sought a family history. However we did not have enough patients with non accommodative, non anisometropic, non infantile esotropia, or high accommodative convergence to accommodation ( $\mathrm{AC} / \mathrm{A}$ ) ratio esotropia to be able to offer any comparison between these types of squint and those found in our patients. Nevertheless, our findings do show that there is a stronger genetic element in hypermetropic accommodative esotropia than in anisometropic esotropia, infantile esotropia, or exotropia in childhood, and that the role of heredity in the etiology of accommodative esotropia deserves further investigation.
2 Schlossmann A, Priestley BS. Role of heredity in etiology and treatment of strabismus. Arch Ophthalmol 1952; 47: 120.

3 Aurell E, Norsell K. A longitudinal study of children with a family history of strabismus: factors determining the incidence of strabismus. Br J Ophthalmol 1990; 74: 589594.

4 Dufier JL, Briard ML, Bonaiti C, Frezal J, Saraux H. Inheritance in the etiology of convergent squint. Ophthalmologica 1979; 179: 225-234.

5 Cohn H. Uber Vererbung und Behandlung des EinwartsSchielen. Berl Klin Wochenschr 1904; 41: 1047-1051.

6 Breslow NE, Clayton DG. Approximate inference in generalized mixed models. J Am Stat Assoc 1993; 88: 9-25.

7 Payne RW, Arnold GM, Morgan GW. Genstat 5 Procedure Library Manual Release 3[1]. Lawes Agricultural Trust: Rothamsted, 1993.

8 Gover M, Yankey JB. Physical impairments of members of low-income farm families-11490 persons. Publ Health Rep 1944; 59: 1163-1184.

9 Frandsen AD. Occurrence of squint. Acta Ophthalmol 1960; 62 (Suppl): 1-122.

10 Nordlow W. Squint: the frequency of onset at different ages and the incidence of some associated defects in a Swedish population. Acta Ophthalmol 1964; 42: 1015-1037.

11 Waardenburg PJ. Squint and heredity. Doc Ophthalmol 1954; 7: 422-494.

12 Czellitzer A. Wie vererbt sich Schielen? Arch Rassen Gesellsch Biol 1923; 14: 377-394.

13 Cross HE. The heredity of strabismus. Am Orthop J 1975; 25: 11-17.

14 Hegmann JP, Mash AJ, Spivey BE. Genetic analysis of human visual parameters in populations with varying incidences of strabismus. Am J Hum Genet 1974; 26: 549562.

\section{References}

1 Hippocrates: Airs, waters and places. In: The Genuine Works of Hippocrates. William Wood \& Co: New York, 1886, p 171. 